\title{
Infecciones graves por Staphylococcus aureus: características clínicas, sensibilidad antibiótica y uso de antimicrobianos. Serie de casos
}

\author{
Severe Staphylococcus aureus infections: clinical characteristics, antibiotic \\ susceptibility and use of antimicrobials. Case series
}

\author{
Dr. Juan Eduardo Ves Losada ${ }^{a}$, Dra. Ana Paula Graziano ${ }^{a}$, Dr. Maximiliano De Abreu ${ }^{b}$, Bioq. Miriam Blanco ${ }^{c}$, \\ Farm. Lorena Frutos ${ }^{d}$, Dr. Lucas Tulae, Dr. Cesar Montali y Dr. Pablo García Munitis ${ }^{a}$
}

\begin{abstract}
RESUMEN
La aparición de infecciones por Staphylococcus aureus (SA) meticilino-resistente (MR) de la comunidad es un problema de salud pública de variable magnitud en diferentes áreas geográficas del mundo. El debate acerca del tratamiento antibiótico empírico inicial permanece abierto.

Nuestro objetivo es presentar una serie de pacientes hospitalizados con infecciones graves por Staphyloccocus aureus en la Unidad de Cuidados Intermedios Pediátricos del Hospital El Cruce.

Entre mayo de 2008 y diciembre de 2010, se internaron 43 pacientes. El 69,8\% de los SA aislados fueron MR. Aunque la diferencia no fue estadísticamente significativa, las infecciones por SAMR requirieron mayor cantidad de días de internación en terapia intensiva y desarrollaron más focos secundarios de infección. Vancomicina, rifampicina y clindamicina fueron los antibióticos más comúnmente prescritos. Existió una alta resistencia de los SA a los $\beta$-lactámicos en la serie estudiada. Palabras clave: Staphylococcus aureus, meticilino-resistente, comunidad, niños.
\end{abstract}

\begin{abstract}
Infectious due to methicillin-resistant community acquired Staphylococcus aureus is a public health problem of varying magnitude in different geographical areas of the world, representing a major burden on health systems. The debate on the initial empiric antibiotic therapy remains open. We present a case series of hospitalized patients with severe Staphylococcus aureus infections admitted to the pediatric intermediate care unit of Hospital El Cruce. Between May 2008 and December 2010, 43 patients were admitted. Sixty nine percent of isolated Staphylococcus aureus were methicillin-resistant. Although the difference was not statistically significant, methicillinresistant Staphylococcus aureus infections required more days of hospitalization in critical care unit and developed more
\end{abstract}

a. Cuidados Intermedios Pediátricos.

b. Investigación.

c. Microbiología.

d. Farmacia.

e. Infectología.

Hospital El Cruce de Florencio Varela.

Correspondencia:

Juan Eduardo Ves Losada: jveslosada@hotmail.com

Conflicto de intereses: ninguno que declarar.

Recibido: 20-11-2013

Aceptado: 7-3-2014 secondary sites of infection. Vancomycin, rifampicin and clindamycin were the most commonly prescribed antibiotics. There was a high resistance of Staphylococcus aureus to $\beta$-lactams in the series.

Keys words: Staphylococcus aureus, methicillin-resistant, community-acquired, children.

http:/ /dx.doi.org/10.5546/aap.2014.e152

\section{INTRODUCCIÓN}

Las infecciones causadas por Staphylococcus aureus meticilino-resistente proveniente de la comunidad (SAMRco) son un problema de salud pública de variable magnitud en diferentes áreas geográficas del mundo, lo que representa una importante carga para los sistemas de salud. ${ }^{1-4}$

En la última década, la prevalencia de este organismo ha aumentado considerablemente en la población general. ${ }^{3-5}$

Un estudio multicéntrico realizado en Argentina reportó que los SAMRco superaron el $58 \%$ en la mayoría de los centros. ${ }^{6}$

No se conoce, en nuestra área de influencia, cuál es el agente antimicrobiano elegido para el tratamiento de las infecciones por SAMR en niños hospitalizados y si los antibióticos beta-lactámicos han sido suplantados por antibióticos con actividad anti-SAMR en el tratamiento empírico inicial de infecciones graves por Staphyloccocus aureus.

Nuestro objetivo es presentar una serie de pacientes hospitalizados con infecciones graves por SA en la Unidad de Cuidados Intermedios Pediátricos (UCIP) del Hospital El Cruce (HEC).

\section{CASOS CLÍNICOS}

El HEC funciona como un centro de derivación, sin consulta externa de demanda espontánea. Los pacientes ingresan, en la mayoría de los casos, por derivación desde otros seis centros hospitalarios de segundo nivel pertenecientes a una red pediátrica de reciente creación o desde otros hospitales de la provincia $\mathrm{u}$ otras regiones del país. La red abarca cuatro 
municipios, donde viven aproximadamente 2000000 de personas. ${ }^{7}$

Se describe una serie de casos clínicos que incluyó a todos los niños de un mes a 16 años, con una infección grave por SA clínica y microbiológicamente documentada, que fueron admitidos en la UCIP del HEC durante el período comprendido entre el $1^{\circ}$ de mayo de 2008 y el 31 de diciembre de 2010.

Se analizaron las características clínicas generales de los pacientes, el uso de antibióticos y el patrón de resistencia.

Se definió infección grave aquella que requirió internación.

Se consideró infección por SA proveniente de la comunidad en los siguientes casos:

a. Cuando el diagnóstico de infección clínica y microbiológicamente documentada se efectuó en las primeras $48 \mathrm{~h}$ de hospitalización o en forma ambulatoria.

b. Cuando el aislamiento bacteriológico se obtuvo de hemocultivos o de una lesión aparente al momento del ingreso y que motivó la internación.

c. Cuando no existieron antecedentes de una admisión hospitalaria anterior en los últimos 12 meses.

Se consideró infección por SA intrahospitalario cuando el diagnóstico de infección clínica y microbiológica se efectuó luego de haber estado $48 \mathrm{~h}$ hospitalizado.

Los antibióticos que se consideraron para el análisis fueron vancomicina, clindamicina, rifampicina, cotrimoxazol, ceftriaxona, cefalotina, teicoplanina y linezolid.
El uso de cada antibiótico se evaluó mediante dos medidas:

a. El porcentaje de uso: se definió como la proporción de pacientes que recibieron uno de los antibióticos especificados en cualquier momento durante su hospitalización (esta medida asignó el mismo peso a un día de antibiótico o a varios días de tratamiento, es decir, fue independiente de la duración de la terapia).

b. Días de terapia (DDT) por 1000 días/paciente: un día de tratamiento se definió como la recepción de $\geq 1$ dosis en un momento dado, por lo que se consideró que el paciente recibió un día de terapia independientemente del número de dosis.

Ingresaron 43 pacientes con diagnóstico de infección grave por SA entre mayo de 2008 y diciembre de 2010.

Las características basales de la población se muestran en la Tabla 1.

Las infecciones por SAMR superaron en frecuencia a las meticilino-sensibles (MS), y se constataron $30(69,8 \%)$ infecciones por SAMR y $13(30,2 \%)$ por SAMS.

Los pacientes con SAMR requirieron más días de internación en Terapia Intensiva que los pacientes con SAMS, aunque la diferencia no fue significativa. Requirieron una intervención 35/43 pacientes $(81 \%)$, sin diferencias entre grupos (Tabla 1).

De los 30 pacientes con infección por SAMR, 7 $(24 \%)$ presentaron el foco inicial en piel y partes blandas, y $5(17 \%)$, un foco pleuropulmonar.

$\mathrm{Al}$ evaluar el porcentaje de uso de antibióticos

TABLA 1. Características generales de los pacientes con infecciones graves por Staphylococcus aureus

\begin{tabular}{lccc}
\hline Variable & SAMS $\mathbf{n = 1 3}$ & SAMR $\mathbf{n = 3 0}$ & $\mathbf{P}$ \\
\hline Edad en años (mediana e IIC) & $3,2(0,5-9,4)$ & $3,3(0,9-9,2)$ & 0,7 \\
Sexo masculino n (\%) & $10(76,9)$ & $15(50)$ & 0,09 \\
SAco n (\%) & $11(84)$ & $27(90)$ & 0,5 \\
Días de internación en sala (mediana e IIC) & $8,5(6-31)$ & $12,5(6-22)$ & 0,74 \\
Días de terapia intensiva (mediana e IIC) & $8,5(5-11)$ & $15(6-34)$ & 0,16 \\
Días totales de internación (mediana e IIC) & $14(10-28)$ & $20,5(10-42)$ & 0,48 \\
Intervenciones & $11(84)$ & $24(80)$ & 0,63 \\
Fallecidos & 1 & 0 & NC \\
\hline
\end{tabular}

SAMS: Staphylococcus aureus meticilino-sensible. SAMR: Staphylococcus aureus meticilino-resistente. NC: no calculable.

IIC: intervalo intercuartilo. SAco: Staphylococcus aureus de la comunidad. 
al momento del ingreso (tratamiento empírico inicial), los antibióticos $\beta$-lactámicos (ceftriaxona y cefalotina) fueron utilizados en el 30\% de los casos, seguidos por la vancomicina, clindamicina, rifampicina y cotrimoxazol en $24 \%, 14 \%, 5 \%$ y $4 \%$ de los casos, respectivamente. Cuando las medidas de uso se utilizaron para los días totales de antibiótico durante la internación, los antibióticos anti-SAMR fueron utilizados en mayor proporción que los $\beta$-lactámicos, teniendo en cuenta tanto el porcentaje de uso como los DDT por 1000 días / paciente (Tabla 2).

Las cepas de SAMRco aisladas presentaron elevada resistencia a la clindamicina $(19 \%)$, mientras que la resistencia al cotrimoxazol fue de 3,4\% (Tabla 3). Los pacientes con infecciones graves por SAMS no presentaron resistencia a los antibióticos comúnmente utilizados.

\section{DISCUSIÓN}

La aparición de cepas de SAMRco en nuestro medio ha sido descrita a partir del año 2003 y ha ido creciendo en forma permanente. ${ }^{3,6,8}$

En nuestro estudio, la tasa de resistencia a la meticilina en los SAMRco fue elevada; alcanzó el $56 \%$ del total de los SA, cifra similar a la hallada por el grupo de Paganini en 2008. ${ }^{6}$

La edad media de los niños infectados por SAMR en nuestro estudio fue de 4,7 años, algo menor a lo descrito en la literatura internacional. ${ }^{9-11}$

La mortalidad de los niños con infección por SA fue del $2 \%$, cifras similares a las comunicadas por Kaplan y Paganini. ${ }^{6,9}$

TABla 2. Medidas de uso de antibióticos en pacientes con infección grave por Staphylococcus aureus

\begin{tabular}{lcc}
\hline Antibiótico & DDT x 1000 días/paciente & Uso (\%) \\
\hline Vancomicina & 51,66 & 60 \\
Rifampicina & 38,14 & 44 \\
Clindamicina & 26,59 & 40 \\
Ceftriaxona & 7,629 & 30 \\
Cotrimoxazol & 11,66 & 22 \\
Cefalotina & 9,045 & 22 \\
Teicoplanina & 4,359 & 10 \\
Linezolid & 5,449 & 4 \\
\hline
\end{tabular}

DDT x 1000 días/paciente: días de terapia por

1000 días/paciente.
Las primeras infecciones causadas por SAMR de la comunidad informadas internacionalmente tenían curso benigno, y prevalecían las infecciones con comienzo en piel y partes blandas. En los últimos años, aumentaron los informes de casos de infecciones graves con compromiso pulmonar inicial, coincidente con nuestra serie. ${ }^{8-12}$

El alto porcentaje de foco pulmonar descrito en nuestros pacientes puede atribuirse al sesgo de selección, por tratarse de una serie de pacientes derivados a Unidades de Terapia Intensiva e Intermedia Pediátricas, con una gravedad en estos casos también reflejada en variables, como los días de internación en Terapia Intensiva y la frecuente necesidad de resolución quirúrgica. ${ }^{6,8,13}$

Los antibióticos más utilizados en el tratamiento empírico inicial de nuestra serie fueron los $\beta$-lactámicos. Como era de esperar por la alta prevalencia de SAMR, cuando se calcularon el porcentaje de uso y los DDT por 1000 días / paciente durante la internación, vancomicina, rifampicina, clindamicina y cotrimoxazol fueron los antibióticos más utilizados. Aunque la sensibilidad a la rifampicina no fue reportada por otros autores, resultó ser un antibiótico comúnmente utilizado en nuestro medio para tratar las infecciones graves por SA. ${ }^{6,8,11,14}$

No se registraron cepas resistentes a vancomicina en nuestro estudio. La vancomicina continúa siendo el tratamiento de elección para las infecciones causadas por SAMR, pero su distribución tisular limitada, principalmente en el pulmón, y la emergencia de cepas con sensibilidad reducida y resistencia in vitro han

TABla 3. Resistencia a los antibióticos de las cepas de Staphylococcus aureus meticilino-resistente aisladas

\begin{tabular}{lcc}
\hline Antibiótico & SAMRco $\mathbf{n = 2 7}$ & SAMRih $\mathbf{n = 3}$ \\
\hline Rifampicina & 1 & 0 \\
Ciprofloxacina & 1 & 1 \\
Cotrimoxazol & 1 & 0 \\
Clindamicina & 5 & 1 \\
Minociclina & 0 & 0 \\
Vancomicina & 0 & 0 \\
\hline
\end{tabular}

SAMRco: Staphylococcus aureus meticilino-resistente de la comunidad.

SAMRih: Staphylococcus aureus meticilino-resistente intrahospitalario. 
forzado la aparición de nuevos agentes. ${ }^{14} \mathrm{La}$ vancomicina debería utilizarse en pacientes con infecciones graves, particularmente con necrosis, cuando exista una incidencia de SAMR en la comunidad superior al $15 \%$ y en pacientes con celulitis y contacto múltiple con sistemas de salud. ${ }^{15}$

Según la recomendación de expertos, la clindamicina es un antibiótico útil para el tratamiento empírico de estas infecciones cuando la tasa de resistencia es inferior al 15\% en el área de trabajo. ${ }^{6}$ La tasa de resistencia en nuestros pacientes fue de $19 \%$ para las infecciones por SAMR de la comunidad. Aunque el número de pacientes fue escaso como para desaconsejar su uso, sugerimos, en nuestra área de trabajo, la elección de una alternativa terapéutica hasta tener el resultado del antibiograma y remarcamos la necesidad de una vigilancia estrecha y permanente de los perfiles de resistencia locales.

A pesar de las limitaciones de nuestro estudio (bajo número de casos y sesgo de selección por tratarse de pacientes derivados a Terapia Intensiva e Intermedia), que pueden restarle validez externa, consideramos de gran utilidad nuestro relevamiento para generar estrategias terapéuticas efectivas y orientadas al uso racional de antibióticos en nuestra área de influencia.

La presentación clínica inicial mostró un predominio de infecciones de piel y partes blandas y pulmonares. Los $\beta$-lactámicos fueron el grupo de antibióticos más utilizados como esquema empírico inicial. Vancomicina, rifampicina y clindamicina fueron los antimicrobianos más comúnmente prescritos en los pacientes con infección grave por SA. Existió una alta resistencia del Staphylococcus aureus a los $\beta$-lactámicos en nuestra serie.

\section{BIBLIOGRAFÍA}

1. Paganini H. Staphilococcus Aureus. En: Paganini H, ed. Infectología Pediátrica. Buenos Aires: Científica Interamericana; 2007.Págs.955-61.

2. Todd J. Staphilococcus Aureus. En: Kliegman R, Stanton B, St. Geme J, Schor N, Behrman R, eds. Nelson. Tratado de

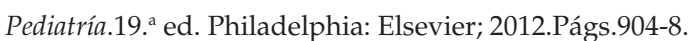

3. Laupland KB, Ross T, Gregson DB. Staphylococcus aureus bloodstream infections: risk factors, outcomes, and influence of methicillin resistance in Calgary, Canada, 2000-2006. J Infect Dis 2008;198(3):336-43.

4. Böcher S, Gervelmeyer A, Monnet DL, Molbak K, et al. Methicillin-resistant Staphylococcus aureus: risk factors associated with community-onset infections in Denmark. Clin Microbiol Infect 2008;14(10):942-8.

5. Chambers H. The changing epidemiology of Staphylococcus aureus? Emerg Infect Dis 2001;7(2):178-82.

6 Paganini H, Della Latta MP, Muller Opet B, Ezcurra G, et al. Estudio multicéntrico sobre las infecciones pediátricas porStaphylococcus aureus meticilino-resistente provenientes de la comunidad en la Argentina. Arch Argent Pediatr 2008;106(5):397-403.

7. García Munitis P, De Abreu M, Antonietti L, Guillen M, et al. Sistema de referencia-contrarreferencia entre el segundo y el tercer nivel de salud en una red hospitalaria pediátrica en el conurbano bonaerense. Arch Argent Pediatr 2013;111(5):405-11.

8. Paganini H, Della Latta P, Soto A, Casimir L, et al. Bacteriemias por Staphylococcus aureus adquiridas en la comunidad: 17 años de experiencia en niños dela Argentina. Arch Argent Pediatr 2010;108(4):311-7.

9. Herigon JC, Hersh AL, Gerber JS, Zaoutis TE, Newland JG. Antibiotic management of Staphylococcus aureus infections in US children's hospitals, 1999-2008. Pediatrics 2010;125(6);e1294-300.

10. SattlerCA,MasonEOJr,KaplanSL.Prospective comparison of risk factors and demographic and clinical characteristics of community-acquired, methicillin-resistant versus methicillin-susceptible Staphylococcus aureus infection in children. Pediatr Infect Dis J 2002;21(10):910-7.

11. StryjewskiME,Chambers HF. Skin and soft-tissueinfections caused by community-acquired methicillin-resistant Staphylococcus aureus. Clin Infect Dis 2008;46Suppl5:S368-77.

12. Chen AE, Carroll KC, Diener-West M, Ross T, et al. Randomized controlled trial of cephalexin versus clindamycin for uncomplicated pediatric skin infections. Pediatrics 2011;127(3);e573-80.

13. Vidal PM, Trindade PA, Garcia TO, Pacheco RL, et al. Differences between "classical" risk factors for infections caused by methicillin-resistant Staphylococcus aureus (MRSA) and risk factors for nosocomial bloodstream infections caused by multiple clones of the staphylococcal cassette chromosome mec type IV MRSA strain. Infect Control Hosp Epidemiol 2009;30(2):139-45.

14. Micek ST. Alternatives to vancomycin for the treatment of methicillin-resistant Staphylococcus aureus infections. Clin Infect Dis 2007;45(Suppl 3):S184-90.

15. Stevens DL, Bisno AL, Chambers HF, Everett ED, et al. Practice guidelines for the diagnosis and management of skin and soft-tissue infections. Clin Infect Dis 2005;41(10):1373-406. 